Conclusion In healthy adults pneumococcal colonisation is an asymptomatic event, regardless of density or viral cocolonisation.

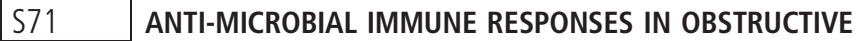 LUNG DISEASES}

${ }^{1} \mathrm{~F}$ Jaat, ${ }^{1} \mathrm{~S}$ Hasan, ${ }^{1} \mathrm{C}$ Lanyon, ${ }^{2} \mathrm{~A}$ De Soyza, ${ }^{1} \mathrm{~S}$ Todryk. ${ }^{1}$ Northumbria University, Newcastle Upon Tyne, UK; ${ }^{2}$ Freeman Hospital, Newcastle Upon Tyne, UK

\subsection{6/thoraxjnl-2015-207770.77}

Background People with chronic lung diseases, such as Bronchiectasis (BE) and smoking-induced Chronic Obstructive Pulmonary Disease (COPD), are susceptible to lung infections which can exacerbate their disease and can be life threatening. A relatively limited range of pathogens cause infections in these conditions and patients suffer repeated infections. It is unclear why such infections do not elicit protective adaptive immune responses. We wish to better understand immune responses against lung-infecting microbes in people with underlying lung disease since immune responses may be connected to disease pathology and also to protection from infection, and may provide a useful marker of colonisation.

Methods We took peripheral blood samples from $114 \mathrm{BE}$ and 47 COPD patients attending secondary care clinics, and 25 healthy controls, and extracted PBMC and serum. The patients were well-characterised clinically, including their history, aetiology, lung function and longitudinal microbial colonisation. T cell and antibody responses were measured against a panel of common lung-infecting microbial antigens (bacteria, fungi and viruses) using our in-house well-characterised assays (ELISA and ELIspot, respectively). These provided quantitative outputs of specific antibody titre and reactive gamma-interferon-secreting $\mathrm{T}$ cells per million PBMC, validated using positive controls. The sputum of patients was cultured, and microbial colonisation defined using prior definitions. Correlations between culture status and bacterial immune responses were analysed.

\begin{tabular}{|l|r|r|}
\hline \multicolumn{1}{|c|}{ Abstract S71 Table 1 } & \multicolumn{1}{l|}{ COPD } \\
\hline P.aeruginosa & 52.6 & 14.8 \\
\hline H.influenzae & 53.5 & 40.4 \\
\hline A. fumigatus & 23.6 & 4.2 \\
\hline M. catarrhalis & 39.4 & 29.7 \\
\hline S.pneumoniae & 47.3 & 21.2 \\
\hline S.maltophilia & 16 & 4.2 \\
\hline Candida & 15.7 & 8.5 \\
\hline S.aureus & 31.5 & 2.1 \\
\hline E.coli & 21 & 6.3 \\
\hline
\end{tabular}

Results The predominant pathogens varied between BE and COPD as expected (percentages in Table 1). These included
Pseudomonas, Haemophilus influenzae, Streptococcus pneumoniae and Moraxella $s p p$. We found that specific $\operatorname{IgG}$ antibody responses correlated with bacterial sputum culture data for Pseudomonas $(\mathrm{R}=0.61, \mathrm{p}=0.0001)$, but not with lung function nor number of exacerbations. In contrast, specific $\mathrm{T}$ cell responses did not correlate with microbiology.

Conclusions Our findings suggest that immune responses measured in the blood against potential lung pathogens contribute minimally to protection from infection or pathology. These tests may however help define colonisation status and could be used as surrogate markers of pathogens in the lung. The poor correlation between $\mathrm{T}$ cell responses may be a facet of the disease.

\section{Improving lung cancer outcomes}

\section{S72 IMPROVING LUNG CANCER SURVIVAL IN ENGLAND EVIDENCED THROUGH MULTIPLE DATA SOURCES}

${ }^{1} \mathrm{P}$ Beckett, ${ }^{1}$ I Woolhouse, ${ }^{2} \mathrm{~S}$ Walters, ${ }^{2} \mathrm{~S}$ Benitez-Majano, ${ }^{2} \mathrm{P}$ Muller, ${ }^{3} \mathrm{D}$ West, ${ }^{4} \mathrm{~S}$ McPhail, 4J Broggio, 'MD Peake. 'Royal College of Physicians, London, UK; ${ }^{2}$ Cancer Research UK Cancer Survival Group, London School of Hygiene and Tropical Medicine, London, UK; ${ }^{3}$ Society of Cardiothoracic Surgeons, Great Britain and Ireland; ${ }^{4}$ NHS Cancer Improvement, National Cancer Intelligence Network, London, UK

\subsection{6/thoraxjnl-2015-207770.78}

Introduction We have collated data from several sources to demonstrate that efforts made over the last 10 years to use data to drive service improvement and improved patient outcomes for UK patients with lung cancer have been successful.

Methods We used data submitted to the National Lung Cancer Audit (NLCA), National Cancer Registration Service (NCRS), Office for National Statistics (ONS) and Society of Cardiothoracic Surgeons (SCTS) from 1995-2013. We calculated numbers and proportions undergoing surgery, case-mix adjusted hazard ratios for death, and actual and predicted (using hybrid analyses) 1-year and 5-year survival for lung cancer patients in England. An international comparison has been made using data from the CONCORD-2 study.

Results In the NLCA, the proportion of NSCLC patients undergoing resection has risen from $14 \%$ (2005) to $23 \%$ (2013). Over this period, annual primary lung cancer resections have risen from 3,220 to 6,713 .

NLCA data, adjusted for age, sex, stage and PS, indicates a gradually falling hazard ratio for death (2013 HR 0.87, 95\% CI 0.85-0.89 compared to 2008). ONS data demonstrates a gradual improvement in both $1 \mathrm{yr}$ and $5 \mathrm{yr}$, and mirrors the increase in the number of resections carried out over the lifetime of the NLCA. Comparison of 1YS with other countries suggests that England has passed the survival measured in Denmark in 200407 (35\%), but still lags behind Canada (42\%) and Sweden $(46 \%)$. In another analysis using NCRS data, comparing stagespecific 1 yr survival in England in 2004-07 and 2012, improvements are most marked in patients with early stage disease.

Conclusion Whilst many changes have taken place in the management of lung cancer over the last 10 years, the close temporal association between the date of the first NLCA report (2005), the numbers of resections carried out and the significant improvements in 1 and $5 \mathrm{yr}$ survival (weighted towards earlier stages) and mortality we report here, would strongly suggest that the NLCA has been successful in its aim to improve standards of care and outcomes for patients. These improvements in survival 
bring England close to parity with other westernised countries, though there is still more work to do.

Abstract S72 Table 1 Resections, actual/predicted survival and incremental survival improvements over study period

\begin{tabular}{|c|c|c|c|c|c|}
\hline Year & $\begin{array}{l}\text { Number of } \\
\text { resections }\end{array}$ & $\begin{array}{l}1 \mathrm{yr} \\
\text { survival } \\
\%\end{array}$ & $\begin{array}{l}5 \text { yr survival (\%) } \\
\text { (actual or } \\
\text { predicted }^{*} \text { ) }\end{array}$ & $\begin{array}{l}\text { Improvement } \\
\text { in } 1 \mathrm{yr} \\
\text { survival }\end{array}$ & $\begin{array}{l}\text { Improvement } \\
\text { in } 5 \mathrm{yr} \\
\text { survival }\end{array}$ \\
\hline 1995 & \multirow{11}{*}{$\begin{array}{l}3220 \\
\text { (mean) }\end{array}$} & 23.2 & 6.5 & \multirow{5}{*}{$+0.73 \% / y r$} & \multirow{5}{*}{$+0.22 \% / y r$} \\
\hline 1996 & & 23.3 & 6.7 & & \\
\hline 1997 & & 25.1 & 7.4 & & \\
\hline 1998 & & 25.0 & 7.2 & & \\
\hline 1999 & & 26.1 & 7.4 & & \\
\hline 2000 & & 27.4 & 8.0 & \multirow{6}{*}{$+0.48 \% / y r$} & \multirow{6}{*}{$+0.12 \% / y r$} \\
\hline 2001 & & 27.7 & 8.2 & & \\
\hline 2002 & & 28.0 & 8.0 & & \\
\hline 2003 & & 29.2 & 9.1 & & \\
\hline 2004 & & 29.2 & 8.5 & & \\
\hline 2005 & & 30.3 & 9.1 & & \\
\hline 2006 & 3740 & 30.1 & 9.1 & \multirow{4}{*}{$+0.79 \% / y r$} & \multirow{4}{*}{$+0.65 \% / y r$} \\
\hline 2007 & 4100 & 32.2 & 10.3 & & \\
\hline 2008 & 4350 & 32.5 & 10.8 & & \\
\hline 2009 & 4500 & 33.5 & $11.8^{*}$ & & \\
\hline 2010 & 5250 & 34.8 & $12.7^{*}$ & \multirow{4}{*}{$+2.15 \% / y r$} & \multirow{4}{*}{$+1.05 \% / y r$} \\
\hline 2011 & 6360 & 36.3 & $13.8^{*}$ & & \\
\hline 2012 & 6474 & 39.0 & $14.9^{*}$ & & \\
\hline 2013 & 6713 & $\mathrm{n} / \mathrm{a}$ & $16.0^{*}$ & & \\
\hline
\end{tabular}

\section{S73 HEALTHCARE COSTS ASSOCIATED WITH LUNG CANCER} DIAGNOSED AT EMERGENCY HOSPITALISATION

${ }^{1}$ MPT Kennedy, ${ }^{2}$ PS Hall, ${ }^{1}$ MEJ Callister. 'Leeds Teaching Hospitals NHS Trust, Leeds, UK; ${ }^{2}$ Edinburgh Cancer Research Centre, University of Edinburgh, Edinburgh, UK

10.1136/thoraxjnl-2015-207770.79

Introduction The diagnosis of lung cancer at emergency presentation with hospital admission is a poor patient experience and is likely to incur significant cost. Reducing the proportion of patients diagnosed by this route has been identified as a priority by policy makers. The full health-economic impact of this route to diagnosis is not known.

Aim To measure the excess healthcare costs attributable to emergency hospital admission in patients diagnosed with lung cancer.

Methods Retrospective review of an electronic database of lung cancer patients from 2008-2013. Secondary care costs were acquired from the local NHS Patient Level Information and Costing System (PLICS) with adjustment for inflation. To adjust for survival differences, secondary analyses looked at average costs incurred only while patients remained alive.

Results 3,274 consecutively diagnosed patients were included. Mean one and twelve-month costs were $£ 2,400 \quad(95 \% \mathrm{CI}$ $£ 2,313-£ 2,493)$ and $£ 10,009$ (95\% CI $£ 9,725-£ 10,285)$. One month mean costs for patients with emergency admission were higher than for those diagnosed by other routes $(£ 3,499$ (95\% CI $£ 3,332-£ 3,667)$ vs $£ 1,899$ (95\% CI $£ 1,810-£ 1,999)$ ). Twelve month mean costs for emergency admissions were lower than for other routes $(£ 8,123(95 \%$ CI $£ 7,704-£ 8,552)$ vs $£ 10,870$ (95\% CI $£ 10,511-£ 11,211)$ ), but this analysis is heavily influenced by excess mortality within the emergency admission group ( 1 year survival $14 \%$ vs $50 \%$ respectively).

Mean costs for survival, only considering costs per patient alive in that month, are shown in Figure 1. Emergency admission was associated with increased mean alive costs compared to other routes at both one month $(£ 3,499$ vs $£ 1,899)$ and 12 months ( $£ 15,063$ vs $£ 13,233)$. Adjusted costs accrued between one and twelve months following diagnosis were similar between the two groups ( $£ 11,565$ vs $£ 11,334)$.

Conclusion Patients diagnosed with lung cancer during an emergency admission incur greater healthcare costs during the first month following diagnosis. Lower longer term costs in these patients seem to be entirely due to the lower survival rates in this poor prognosis group.

In addition to improving patient experience and outcome, strategies to increase earlier diagnosis of lung cancer may reduce the additional healthcare costs associated with this route to diagnosis.

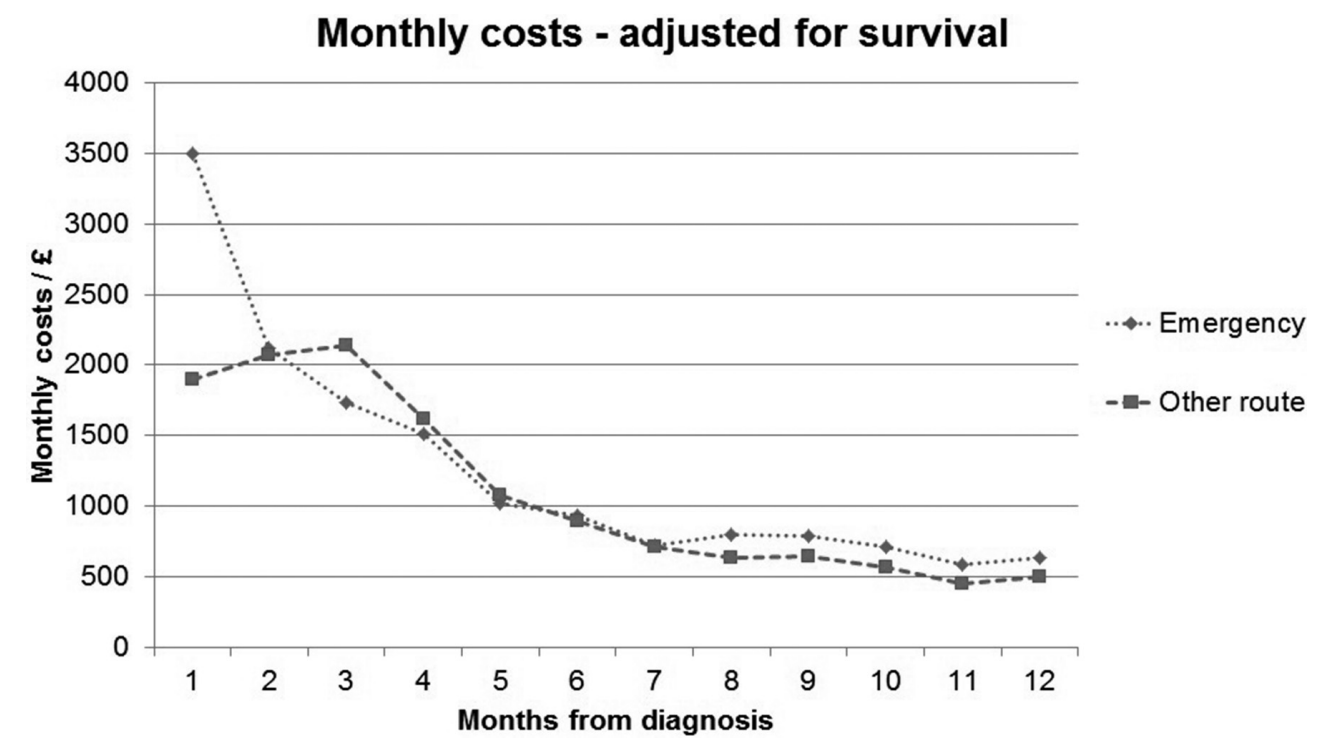

Abstract S73 Figure 1 